\title{
Лимфангиолейомиоматоз: обзор литературы
}

ГОУ ВПО "ММА им. И.М.Сеченова": 119991, Москва, ул. Трубецккая, 8, стр. 2

\section{S.I.Ovcharenko, E.A.Son \\ Lymphangioleiomyomatosis (review)}

Key words: lymphangioleiomyomatosis, diagnosis, tuberous sclerosis, lymphangioleiomyoma, chylous pleural effusion, pneumothorax. Ключевые слова: лимфангиолейомиоматоз, диагностика, туберозный склероз, лимфангиолейомиома, хилезный плевральный выпот, пневмоторакс.

Лимфангиолейомиоматоз (ЛАМ) - редкое заболевание с поражением легких и лимфатической системы, встречающееся почти исключительно у женщин и развивающееся обычно до наступления менопаузы [1]. Морфологическим маркером ЛАМ является обнаружение в пораженных органах специфических для этого заболевания клеток - ЛАМ-клеток. Морфологическим субстратом болезни является мультифокальная узловая пролиферация специфических ЛАМ-клеток, располагающаяся преимущественно по ходу лимфатических сосудов и в лимфатических узлах, сочетающаяся в легких с кистозной перестройкой его паренхимы [2].

Клиническая картина ЛАМ складывается из легочных и внелегочных проявлений. К легочным проявлениям относятся прогрессирующая кистозная перестройка паренхимы легких с развитием рецидивирующих пневмотораксов, хилезного плеврального выпота и формированием в исходе заболевания дыхательной недостаточности [1]. Среди внелегочных проявлений наиболее часто встречаются лимфаденопатия, расширение и утолщение лимфатических сосудов, образующих целые кистозные конгломераты и поэтому называемых "лимфангиолейомиомами", "хилезным асцитом" и "ангиомиолипомами" [1].

Выделяются 2 варианта ЛАМ - спорадический и сочетанный с туберозным склерозом (ТС). СпорадическиЙ ЛАМ и ЛАМ сочетанныЙ с ТС (ЛАМ-ТС) морфологически неразличимы [2]. Впервые спорадический ЛАМ был описан в 1937 г. [3]. Распространенность спорадического ЛАМ составляет 1-2 случая на 1 млн взрослых женщин, или 1 случай на 400 тыс. взрослых женщин [4, 5]. Распространенность же ЛАМ среди больных ТС составляет 30-40 \% взрослых женщин [4-6].

ТС - генетически детерминированное заболевание, наследуемое по аутосомно-доминантному типу и относящееся к группе нейроэктодермальных нарушений. Учитывая мультисистемность поражений при ТС, это заболевание также принято называть туберозно-склерозным комплексом (tuberous sclerosis complex - TSC). ТC, или болезнь Бурневилля-Прингля, - аутосомно-доминантное заболевание, относя- щееся к факоматозам - системным наследственным дисплазиям, связанным с нарушением закладки эктодермального зародышевого листка и характеризующееся комбинированными опухолевидными поражениями кожи, головного мозга, глазных яблок, сердца, почек и легких [7]. Кроме ТС, к группе факоматозов относятся нейрофиброматоз, или болезнь Реклингхаузена, синдром Стержа-Вебера и болезнь Гиппеля-Линдау. Учитывая то, что распространенность ТС, по разным данным, колеблется и составляет от 1 случая на 30 тыс. взрослого населения [8] до 1 случая на 150 тыс. взрослого населения [1], по самым приблизительным подсчетам, ЛАМ-ТС встречается примерно в 3-17 раз чаще, чем спорадический ЛАМ. Поэтому вполне закономерно, что самое первое (в 1918 г.) описание этого заболевания [9] было посвящено именно ЛАМ у больного с ТС, т. е. ЛАМ, ассоциированному с ТС, который значительно чаще встречается в популяции. Однако, несмотря на то, что почти 1/3 пациентов, страдающих ТС, имеют рентгенологические признаки ЛАМ легких, впервые выявленные при компьютерной томография высокого разрешения (КТВР), только у 2-3 \% из них в дальнейшем развивается симптоматика ЛАМ [10, 11].

Учитывая имеющиеся описания случаев, помимо Европы, из Юго-Восточной Азии, Японии и Северной Америки, можно говорить о том, что ЛАМ поражает все этнические группы населения Земли [4, 12-14].

Этиология и патогенез ЛАМ до конца неизвестны, однако, принимая во внимание бо́льшую распространенность этого заболевания среди лиц, страдающих ТС, возникает мысль об их этиологическом и / или патогенетическом сходстве. На родство ЛАМ и ТС указывает наличие инактивирующих мутаций в одних и тех же генах - генах TC (TSC1 и TSC2). Причем мутации в этих генах имеют место как при ЛАМ, ассоциированном с ТС, так и при спорадическом ЛАМ [15]. Однако, если ТС является наследственным заболеванием и наследуется по аутосомно-доминантному типу, в связи с чем для него характерна равная вероятность встречаемости как у мужчин, так и у женщин, то для спорадического ЛАМ характерно 
то, что болеют им только женщины [1] и не описаны случаи передачи заболевания "по наследству" - от матери к дочери [16]. Гены TSC1 и TSC2, по своей сути, являются генами-супрессорами опухолевого роста и кодируют синтез белков гамартина и туберина соответственно [7]. В свою очередь, гамартин-тубериновый комплекс подавляет избыточный клеточный рост через мишени для рапамицина млекопитающих - mTOR (mammalian target of rapamycin). Мутации в генах TSC1 и TSC2 приводят к снижению нормальной активности гаматрин-туберинового комплекса и, следовательно, к уменьшению контроля над пролиферацией клеток, рост которых контролируется посредством этого механизма [7]. Для спорадического ЛАМ более характерна мутация в гене TSC2, которая была выявлена в клетках пациенток, полученных из легких [15], крови, мочи [17] и хилезной плевральной жидкости [18]. Тем не менее мутация гена TSC1 так же была описана у больных спорадическим ЛАМ [19]. Таким образом, патогенез ЛАМ сходен с патогенезом ТС: вследствие мутации генов TSC1 и TSC2 имеет место нарушение регуляции пути метаболизма mTOR $[15,19,20]$. Результатом этого является образование популяции ЛАМ-клеток.

При микроскопическом исследовании ЛАМклетки достаточно вариабельны, но на сегодняшний день по внешнему виду выделяют, как минимум, 2 их субпопуляции: незрелые гладкомышечные и эпителиоидные, а также множество промежуточных форм. Предшественники, а следовательно, и гистологическая принадлежность ЛАМ-клеток неизвестны, поскольку, с одной стороны, они экспрессируют гладкомышечный актин, характерный для миоцитов гладких мышц, а с другой - белок премеланоцита, который выявляется посредством положительной реакции с НМВ-45 (моноклональными антителами Human Melanoma Black-45) и содержат премеланосомы - маркеры дифференцировки меланоцитов. Представительство маркеров меланоцитов может наводить на мысль об их происхождении из нервного гребня, поэтому ЛАМ-клетки, внешне напоминающие эпителиальные, хотя и не имеющие с ними более ничего общего, классифицируются как периваскулярные эпителиоидные клетки [21, 22].

Принимая во внимание тот факт, что основным патогенетическим механизмом прогрессирования ЛАМ является бесконтрольная мультифокальная узловая пролиферация ЛАМ-клеток, а так же "генетическая" общность ЛАМ с ТС - гамартомным, т. е. "опухолевоподобным" заболеванием, совершенно естественно возникает мысль об определенном родстве ЛАМ с опухолевыми процессами. Классическими признаками "злокачественности" являются: 1) наличие автономно пролиферирующего бессмертного клона клеток, эволюционирующего в сторону независимости контроля от организма; 2) способность к инвазивному росту и 3) метастазирование [23].

Сопоставляя эти критерии, характеризующие злокачественные новообразования, с имеющимися на сегодняшний день данными, полученными при наблюдении за пациентами с ЛАМ, можно выявить определенное сходство по всем 3 пунктам:

1. Морфологическим субстратом ЛАМ является опухолевоподобный рост ЛАМ-клеток, до некоторой степени бесконтрольный. ЛАМ-клетки, подобно клеткам многих злокачественных опухолей, экспрессируют сосудистый эндотелиальный ростовой фактор D (Vascular Endothelial Growth Factor-D - VEGF-D) [24], благодаря которому поддерживается ангиогенез в узлах, образованных ЛАМ-клетками.

2. В пользу "инвазивности" роста ЛАМ-клеток свидетельствует повышенное содержание в них сывороточного ответного фактора (Serum Response Factor - SRF) и матриксных металлопротеиназ (ММП). SRF увеличивает экспрессию ММП 2-го (коллагеназа IV) и 14-го (мембраносвязанная ММП) типов, содержание которых при ЛАМ повышается [25, 26]. Как известно, степень инвазивного роста и метастазирования опухолевых клеток определяются их способностью расщеплять компоненты экстрацеллюлярного матрикса, такие как базальные мембраны и компоненты межтканевой стромы (коллаген, эластин, ламинин и т. д.), что достигается с помощью повышенного синтеза ММП [27]. В отличие от многих протеолитических ферментов, способных расщеплять отдельные компоненты экстрацеллюлярного матрикса, ММП разрушают все его структуры, что и позволяет опухолевым клеткам внедряться на территорию других тканей. Кроме того, наряду с увеличением экспрессии ММП, при ЛАМ отмечается снижение уровня тканевых ингибиторов ММП 3-го типа (ТІМР-3). Однако следует отметить, что повышение уровня ММП было выявлено и при других интерстициальных заболеваниях легких, таких как идиопатический легочный фиброз и саркоидоз [28].

3. В пользу теории "доброкачественного метастазирования" ЛАМ [29] свидетельствует тот факт, что ЛАМ-клетки биоптата легких и клетки ангиомиолипомы почки у пациентки с ЛАМ имели общую мутацию, а следовательно, и общее происхождение [15]. Широко известен случай рецидива ЛАМ в донорском легком после трансплантации [30]. Кроме того, клетки с мутацией в гене TSC2 были выделены из крови и мочи [17], а так же хилезной плевральной жидкости больных [18]. Тем не менее следует отметить, что параллельно теории "доброкачественного метастазирования" все перечисленные выше факты некоторыми исследователями трактуются в рамках гипотезы о том, что предшественники ЛАМ-клеток представлены и циркулируют в кровообращении.

Наряду с вышеизложенным, в соответствии с накопленными на сегодняшний день фактами, в этиологии и прогрессировании ЛАМ определенную роль, вероятно, играют женские половые гормоны, которые регулируют транскрипцию многих генов, а так же могут играть роль стимула в пролиферации и миграции ЛАМ-клеток в другие органы и ткани. 
В пользу этого предположения свидетельствуют перечисленные ниже факты:

1. Заболевание развивается почти исключительно у женщин детородного возраста и почти всегда симптоматика дебютирует до наступления менопаузы [31, 32]. Первые симптомы заболевания появляются, как правило, после 30 лет, а средний возраст на момент постановки диагноза обычно составляет 41-43 года [32, 33]. После наступления менопаузы заболевали в основном те женщины, которые находились на заместительной терапии эстрогенами [5, 12].

2. Дебют и обострения заболевания могут быть спровоцированы беременностью и лечением эстрогенами, а после наступления менопаузы или удаления яичников прогрессирование заболевания в ряде случаев замедляется [34-36]. Пневмоторакс более часто развивается во время беременности [12].

3. В дополнение ко всему вышесказанному, примерно в половине случаев при проведении иммуногистохимического исследования на ЛАМ-клетках выявлялись поверхностные PRs (прогестероновые) и ERs (эстрогеновые) рецепторы [37, 38], что так же свидетельствует в пользу гормональной зависимости заболевания. По данным отечественных авторов, экспрессия ЛАМ-клетками PRs и ERs была выявлена в 80 \% случаев [39]. Резюмируя вышеизложенное, следует подчеркнуть, что ЛАМ поражает почти исключительно женщин. На сегодняшний день в литературе представлено описание только 5 случаев ЛАМ у мужчин, которые имели гистологическое подтверждение диагноза. В 3 из них речь шла о ЛАМ-ТС, поскольку имел место диагноз достоверного или вероятного ТС [40, 41]. В 1 случае описывался мезентериальный ЛАМ, диагностированный у мужчины с синдромом Кляйнфельтера [42], и лишь в 1 сообщении был описан случай ЛАМ у мужчины с нормальным кариотипом и без явных генетических доказательств ТС [43].

Таким образом, на основании вышеприведенных данных можно говорить о том, что в патогенезе и прогрессировании ЛАМ на сегодняшний день большое значение придается популяции ЛАМ-клеток, которые обладают фенотипом, способным к бесконтрольному росту, экспрессии гормональных рецепторов, протеаз и факторов роста, включая сосудистый эндотелиальный фактор роста, и обладающие метастатическим поведением.

Пролиферация ЛАМ-клеток имеет место в основном в паренхиме легких и лимфатической системе. В легких изменения носят диффузный характер и проявляются мультифокальной узловой пролиферацией ЛАМ-клеток, которая сочетается с кистозной перестройкой паренхимы, связана с пролиферацией лимфатических сосудов [44] и обычно располагается вокруг них, а так же подплеврально и в альвеолярных септах [2]. Кроме этого, при морфологическом исследовании ткани легких описаны расширенные лимфатические протоки, преимуще- ственно в плевре [45], и образование лимфангиолейомиом осевых (располагающихся вдоль оси туловища) лимфатических сосудов грудной клетки [50]. Следствием таких изменений являются клинические симптомы и синдромы, составляющие легочные проявления заболевания и доминирующие в его клинической картине. Самым частым проявлением поражения легких при ЛАМ, по данным 10 наиболее крупных обзоров, является одышка, встречающаяся в $87 \%$ случаев и иногда ассоциированная со стридорозным дыханием [2]. Вероятно, одышка является следствием нарушения диффузионной способности легких (TLCO), которая у 82 \% пациентов снижается уже на начальных стадиях болезни и / или бронхообструкции, поскольку даже при нетяжелом течении ЛАМ сниженный показатель объема форсированного выдоха за 1-ю с $\left(\mathrm{OФB}_{1}\right)$ имеют $58 \%$ больных [4, 46]. У $65 \%$ больных ЛАМ в течение болезни развивается пневмоторакс [2], причем у 4 \% из них он бывает 2-сторонним [47] и достаточно часто рецидивирующим [12]. При этом обращает на себя внимание тот факт, что пневмоторакс у больных ЛАМ чаще развивается во время беременности [12]. Около $51 \%$ больных ЛАМ предъявляют жалобы на кашель, как правило сухой, но на более поздних стадиях болезни было отмечено присоединение выделения мокроты и учащение повторных респираторных инфекций [2]. Характерной особенностью ЛАМ является частое развитие хилезного плеврального выпота, который выявляется у $28 \%$ больных ЛАМ [2], в то время как по частоте встречаемости в общей структуре выпота в плевральную полость хилоторакс занимает одно из последних мест. Частое развитие хилоторакса в клинической картине ЛАМ при столь редком его развитии вследствие других заболеваний позволило отнести его к критериям диагностики этого заболевания $[1,2]$. Он является следствием обструкции ЛАМклетками лимфатических сосудов грудной клетки грудного протока, лимфатических сосудов плевры или средостения [1], поэтому после аспирации жидкости из плевральной полости хилоторакс при ЛАМ имеет тенденцию быстро рецидивировать [12, 48]. У 22 \% лиц, страдающих ЛАМ, встречается гемофтиз как следствие обструкции ЛАМ-клетками легочных капилляров, а у 7 \% - хилофтиз как проявление нарушения проходимости капилляров легких [32].

Внелегочные проявления ЛАМ включают в себя поражение лимфатической системы (лимфангиолейомиомы и лимфоаденопатия), ангиомиолипомы и менингиомы.

Что касается поражения внелегочной лимфатической системы, то особенно характерным для ЛАМ является поражение в основном крупных осевых лимфатических сосудов брюшной полости, таза и ретроперитонеального пространства, которые становятся утолщенными и расширенными, а в некоторых случаях могут закупориваться ЛАМ-клетками [49, 50]. Наиболее специфическим поражением лимфатической системы у больных ЛАМ служит образование лимфангиолейомиом - скопления кистозно увеличенных и расширенных лимфатических 
сосудов, которое выявляется у $21 \%$ пациентов [51, 52]. Разрыв лимфангиолейомиом в $10 \%$ случаев приводит к образованию хилезного асцита [53]. При схожих изменениях в лимфатических сосудах мочевого пузыря появляется хилурия [54]. Также имеются описания хилоперикарда, хотя отмечается, что он редко бывает клинически значимым [1].

У 40 \% пациентов с ЛАМ выявляется лимфоаденопатия с преимущественным поражением ретроперитонеальных, реже тазовых, лимфатических узлов, а также лимфатических узлов, находящихся позади места прикрепления ножек диафрагмы - ретрокруральных [52]. Как увеличенные конгломераты лимфатических узлов, так и лимфангиолейомиомы могут приводить к появлению малоспецифичной симптоматики со стороны брюшной полости. Клиническими проявлениями лимфоаденопатии брюшной полости и таза обычно являются наличие пальпируемых образований, ощущение дискомфорта в брюшной полости, метеоризм, парестезии и отеки ног. К несколько более специфичным проявлениям относятся синдромы сдавливания внутренних органов, особенно когда тазовые конгломераты сдавливают мочевой пузырь. Однако следует отметить, что эти симптомы не только малоспецифичны, но встречаются у небольшого количества пациентов. Так, из $21 \%$ больных, имевших ангиомиолипомы, симптоматика со стороны брюшной полости наблюдалась в $45 \%$ случаев, т. е. менее чем у половины [50, 51].

Достаточно часто у больных ЛАМ выявляются ангиомиолипомы - доброкачественные опухоли, состоящие из гладкомышечных ЛАМ-клеток, кровеносных сосудов и жира $[52,55,56]$. Ангиомиолипомы имеют место более чем у $50 \%$ пациентов со спорадическим ЛАМ, и выявляются в 69-80 \% случаев у больных ТС [57-60], а среди лиц, страдающих ЛАМ, ассоциированным с ТС, ангиомиолипомы имеют место почти у всех пациентов [61]. Учитывая столь частое распространение ангиомиолипом почек при ЛАМ и генетическое единство ЛАМ-клеток легких и почек, их выявление у пациентов с характерной для ЛАМ КТ-картиной легких является одним из возможных вариантов достоверной диагностики ЛАМ [2]. При спорадическом ЛАМ ангиомиолипомы обычно выявляются в почках, единичные, < 1,5 см в диаметре, как правило, асимптомны [52], тогда как при ЛАМ-ТС ангиомиолипомы часто большие, множественные, 2-сторонние и кроме почек, могут поражать другие органы (печень, селезенка, средостенье) [62-64]. Опухоли $>4$ см, особенно имеющие аневризматические сосуды, склонны к кровоточивости, проявляются как гематурия и гемоперитонеум $[62,65,66]$. Одновременно с этим как при спорадическом, так и при ЛАМ-ТС, кроме ангиомиолипом в почках могут быть выявлены и злокачественные новообразования - почечноклеточная карцинома и недавно описанный у этих больных карциномоподобный вариант ангиомиолипомы [1, 2].

Последним внелегочным проявлением ЛАМ можно считать менингиому, однако, принимая во внимание значительно меньшую частоту ее возник- новения, по сравнению с другими указанными выше проявлениями болезни, возможно, более правильно говорить об ассоциации менингиомы с ЛАМ. У больных ЛАМ как при спорадическом, так и ассоциированном с ТС, имеется повышенный риск развития менингиомы [67]. При проведении магнитно-резонансной томографии (МРТ) головного мозга 250 пациентам с ЛАМ у 3,6 \% (9 человек) была выявлена менингиома, причем у 3 пациентов менингиома сопровождалась соответствующими симптомами [67]. Поскольку в популяции в целом риск развития менингиомы составляет $0,005 \%$, очевидно, что у больных ЛАМ эта опухоль встречается гораздо чаще $(3,6 \%$ vs $0,005 \%$ ) [67].

Несмотря на наличие в клинической картине лимфангиолейомиоматоза столь демонстративных, необычных и редко встречающихся в рутинной врачебной практике особенных симптомов, как хилезный выпот, рецидивирующий пневмоторакс, наличие лимфангиолейомиом, ангиомиолипом почек и менингиом, возникающих почти исключительно у молодых женщин, диагностика этого заболевания представляет большие трудности, связанные не только с невысокой распространенностью заболевания и малой осведомленностью врачей общей практики, но и с отсутствием до недавнего времени четко сформулированных диагностических критериев ЛАМ. В декабре 2009 г. рабочей группой Европейского респираторного общества впервые были опубликованы Клинические рекомендации по диагностике и лечению лимфангиолейомиоматоза, которые были созданы на основании анализа всех описанных на сегодняшний день наблюдений ЛАМ. В соответствии с разработанными диагностическими критериями, диагноз ЛАМ может быть оценен как достоверный, вероятный или сомнительный [2].

\section{Диагностические критерии лимфангиолейомиоматоза} Достоверный ЛАМ

1. "Характерные для ЛАМ легких" или "совместимые с ЛАМ легких" КТ-признаки и биопсия легких, соответствующая морфологическим критериям ЛАМ, или

2. "Характерные для ЛАМ легких" КТ-признаки и любой из нижеследующих:

а) ангиомиолипома (почки);

б) хилезный выпот в грудной или брюшной полости;

в) лимфангиолейомиома или поражение лимфоузлов, характерное для ЛАМ;

г) определенный или возможный ТС.

\section{Вероятный ЛАМ}

1. "Характерные для ЛАМ легких" КТ-признаки и клиническая картина или

2. "Совместимые с ЛАМ легких" КТ-признаки и любой из следующих: ангиомиолипома почки, хилезный выпот в грудной или брюшной полости. 


\section{Сомнительный ЛАМ}

"Характерные для ЛАМ легких" или "совместимые с ЛАМ легких" КТ-признаки

По степени специфичности для ЛАМ рентгенологические критерии подразделены на "характерные для ЛАМ легких" и "совместимые с ЛАМ легких" легких [2].

Характерные для ЛАМ легких КТ-признаки являются более специфичными для ЛАМ и включают множественные тонкостенные круглые хорошо дифференцируемые воздушные кисты при сохранном или увеличенном объеме легких без других значимых поражений, отсутствие признаков других интерстициальных заболеванй легких, за исключением маленьких узелков, которые наиболее часто являются КТ-проявлением мультифокальной микроузловой гиперплазии пневмоцитов у пациентов с ТС (как без, так и в сочетании с ЛАМ) [73].

К совместимым с ЛАМ легких изменениям, выявляемым при КТВР, относят наличие только небольшого количества (от 2 до 10) таких кист (до 30 мм в диаметре), при соблюдении прочих вышеперечисленных условий.

Из оценки диагностической значимости существующих критериев следует, что самыми информативными исследованиями в диагностике ЛАМ являются КТВР и морфологическое исследование ткани легкого, поскольку для этих методов разработаны наиболее достоверные критерии. Сочетание положительных результатов обоих этих исследований позволяют убедительно диагностировать ЛАМ [2].

По данным большинства имеющихся на сегодняшний день исследований, к "золотому стандарту" диагностики относят морфологическое исследование биоптата ткани легкого. Критериями диагноза, на основании наличия которых опытный патологоанатом может диагностировать ЛАМ, служат одновременное присутствие множественных мелких воздушных кист и наличие мультифокальной узловой пролиферации 2 основных видов ЛАМ-клеток незрелых гладкомышечных и периваскулярных эпителиоидных клеток [49, 69, 70]. Поскольку опыт морфолога в диагностике столь редкого заболевания не всегда позволяет сделать однозначные выводы, а так же в случаях, когда морфологическая картина, особенно на ранних стадиях заболевания, не вполне специфична, целесообразно проведение дополнительного иммуногистохимического исследования для выявления экспрессии $\alpha$-гладкомышечного актина и НМВ-45 [46, 68, 71, 72]. Подобное расширение морфологического обследования так же показано в случаях трансбронхиального метода получения образца ткани легкого [68]. Кроме этого, полезным дополнением при постановке морфологического диагноза может быть определение рецепторов к эстрогену и прогестерону [2, 37-39].

Тем не менее, в соответствии с разработанными на сегодняшний день критериями диагностики ЛАМ, достоверный диагноз может быть верифицирован и без проведения исследования биоптата [2].
Распространение в последние годы в клинической практике КТВР позволило выявить рентгенологические маркеры поражения легких при ЛАМ, что сделало этот метод исследования обязательным в диагностике данного заболевания, поскольку на основании убедительных КТ-признаков, в сочетании с соответствующей клинической картиной, ЛАМ можно диагностировать с высокой достоверностью и без проведения биопсии легкого [2]. В тех случаях, когда достоверный диагноз ЛАМ ставится без биопсии легких, помимо результатов КТВР большое значение придается как легочным, так и внелегочным клиническим проявлениям заболевания.

Как указывалось выше, внелегочными критериями диагностики ЛАМ являются наличие ангиомиолипомы почки (подтверждается при наличии КТ - и / или морфологических критериев опухоли), хилезного выпота в плевральной или брюшной полости (характер выпота устанавливается либо визуально, либо по биохимическим маркерам), лимфангиолейомиомы или лимфоаденопатии, характерной для ЛАМ (с обязательной морфологической верификацией) или достоверного или вероятного диагноза ТС, который устанавливается в соответствии с критериями диагноза болезни Бурневилля-Прингля [2]. В соответствии с разработанными критериями диагностики, вероятный диагноз ЛАМ может быть поставлен при сочетании характерных КТ-признаков с соответствующей клинической картиной, включающей (множественный и / или билатеральный пневмоторакс) и / или соответствующие ЛАМ изменения функции легких [2], которые состоят в преимущественном снижении TLCO и в меньшей степени - OФВ 1 , при сохранных легочных объемах [2, 12, 32, 74, 75].

После верификации диагноза ЛАМ для выбора дальнейшей тактики ведения пациента требуется оценка риска возникновения потенциально возможных клинически значимых осложнений заболевания, к которым относятся пневмоторакс, накопление хилезного выпота и развитие кровотечения из имеющихся ангиомиолипом почек. Их появление является закономерным проявлением эволюции и прогрессирования ЛАМ, поэтому пациенты должны быть хорошо осведомлены о возможных симптомах появления этих опасных для жизни осложнений и информированы о необходимости экстренного поиска медицинской помощи в случаях их появления [2]. Дебют пневмоторакса, кровотечения и хилезного выпота может быть связан / спровоцирован 2 основными факторами, к которым относятся беременность и терапия экзогенными эстрогенами [12, 76-84]. Таким образом, учитывая имеющиеся на сегодняшний день данные, пациентам, страдающим ЛАМ, целесообразно разъяснять возможные последствия беременности, рекомендовать воздерживаться от нее, а так же избегать терапии эстроген-содержащими препаратами, включая комбинированные контрацептивные препараты и заместительную гормональную терапию [2].

Кроме этого, некоторую предусмотрительность пациентам с ЛАМ и перенесенным пневмотораксом 
следует соблюдать в отношении совершения авиаперелетов. Принимая во внимание тот факт, что во время полета, при снижении атмосферного давления в кабине самолета, воздух в плевральной полости, при наличии пневмоторакса, расширяется и может привести к переходу обычного пневмоторакса в угрожающий жизни напряженный. Полеты самолетом запрещены пациентам с ЛАМ, имеющим нелеченный пневмоторакс или пневмоторакс пролеченный в течение месяца [2]. Таким образом, пациенты должны быть предупреждены, что при появлении новой респираторной симптоматики, не оцененной врачом, от полета следует воздержаться, поскольку она может быть проявлением пневмоторакса. Данных о повышенном риске развития пневмоторакса у больных ЛАМ при авиаперелетах нет, но имеются сведения о развитии головокружения и гипоксемии, особенно у лиц с нарушениями функции дыхания [85]. В связи с этим пациентам с ЛАМ не следует запрещать путешествовать по воздуху, но лиц с развернутыми стадиями болезни следует оценить на предмет необходимости кислородной поддержки в течение полета [2].

В случаях развития пневмоторакса при ЛАМ, как и при пневмотораксе другого генеза, используются консервативные и хирургические методы лечения. К консервативным относят наблюдение, предполагающее возможность самостоятельной резорбции воздуха из полости плевры; аспирацию посредством плевральной пункции или установки плевральной дренажной трубки с последующим проведением плевродеза. Однако следует отметить, что эти методы у больных ЛАМ ассоциируются с рецидивом пневмоторакса в 2/3 всех случаев [12]. Радикальные хирургические методы лечения, направленные на устранение причин, предотвращение рецидивов и плевропульмональных осложнений пневмоторакса, включают в себя плевродез с видеоторакоскопическим пособием, плевральную абразию, плеврэктомию и буллэктомию.

При развитии хилоторакса рекомендуется назначение безжировой диеты, а при значительном объеме плеврального выпота используются различные процедуры, выбор которых определяется хирургом индивидуально в каждой ситуации [2]. В литературе представлены сведения об использовании торакоцентеза, плевродеза посредством инстилляции склерозирующих веществ или париетальной плеврэктомии, торакотомии с перевязкой грудного протока и плевроперитонеального шунтирования [48, 86-93].

Еще одним частым осложнением ЛАМ является кровотечение из ангиомиолипом. В связи с этим пациенты, имеющие ангиомиолипомы, должны быть осведомлены о возможных симптомах кровотечения и в случае их возникновения - о необходимости экстренного обращения за врачебной помощью [2]. Индикаторами риска кровотечений считаются размер опухоли $>4$ см и наличие в ангиомиолипоме аневризматически расширенных сосудов с аневризмами $\geq 5$ мм, т. к. эти критерии имели место при всех подобных геморрагических поражениях [2, 94].
Поскольку аневризматически измененные сосуды ангиомиолипом хорошо выявляются только при ангиографии почек, больные, имеющие ангиомиолипомы $>4$ см в диаметре, требуют более пристального наблюдения с выполнением дважды в год ультразвукового исследования почек для оценки динамики роста ангиомиолипом [2], с тем, чтобы иметь возможность своевременного проведения ангиографии с последующим обсуждением радикального лечения (резекция или эмболизация) ангиомиолипомы не только при остром кровотечении, но и профилактически.

Кроме наблюдения за пациентами, в настоящее время ведутся активные поиски методов лечения, направленных на улучшение качества жизни, стабилизацию заболевания и предотвращение прогрессирования необратимых изменений, приводящих к инвалидизации и необходимости трансплантации легких. Наиболее исследованными являются патогенетические медикаментозные методы, включающие в себя применение бронходилататоров, ингаляционных глюкокортикостероидов, прогестерона, антиэстрогенных препаратов и ингибиторов m-TOR.

В связи с тем, что у 20-24\% пациентов с ЛАМ выявлен положительный ответ при проведении бронходилатационного теста $[46,95]$, ингаляционные бронходилататоры следует назначать пациентам с ЛАМ и обструкцией воздушного потока, отвечающим на применение ингаляционных бронходилататоров [2].

Принимая во внимание отсутствие данных, подтверждающих эффективность ингаляционных глюкокортикостероидов при ЛАМ, несмотря на теоретически обоснованное и допустимое воздействие этой лекарственной группы на воспаление воздушных путей в виде бронхиолита, имеющееся при ЛАМ, назначение кортикостероидов не вошло в клинические рекомендации.

Патогенетически обоснованное применение прогестерона при терапии ЛАМ в исследованиях не продемонстрировало достоверного клинического эффекта, в связи с этим прогестерон не должен рутинно назначаться таким больным [2]. Только в случае быстрого прогрессирования падения показателей функции легких лечение прогестероном допустимо при динамическом контроле ОФВ 1 и TLCO каждые 3 мес. в течение 1 года с отменой препарата при отсутствии положительной динамики по истечении этого срока [2].

Среди других антиэстрогенных методов лечения исследовались оофорэктомия, тамоксифен и трипторелин - агонист гонадоптопин-релизинг-фактоpa $[5,35,36,74,89,92,93]$. По имеющимся в настоящее время данным, доказательства эффективности этих методов лечения отсутствуют, хотя для терапии трипторелином была выявлена ассоциация с редукцией минеральной костной плотности [96]. В связи с этим гормональные методы лечения, кроме прогестерона, не следует использовать пациентам с ЛАМ [2].

Поскольку мутации в генах TSC1 и TSC2 ассоциированы с активацией m-TOR-пути, вполне обосно- 
ванным представляется назначение ингибиторов m-TOR - сиролимуса и эверолимуса. Результаты 2 исследований по оценке эффективности сиролимуса у пациентов с ЛАМ не однозначны и требуют дальнейшего изучения эффективности этой лекарственной группы $[97,98]$. В связи с этим, терапия сиролимусом пока должна проводиться в рамках будущих клинических исследований или в случаях быстропрогрессирующего течения заболевания, при взвешенной оценке отношения польза-риск и под пристальным динамическим наблюдением [2].

Учитывая отсутствие эффективных методов лечения ЛАМ, при прогрессирующем течении заболевания в финале встает вопрос о трансплантации легких. Показаниями для этого являются III-IV функциональный класс по NYHA с гипоксемией в покое, тяжелые нарушения легочной функции и дееспособность [2].

Кроме того, несмотря на отсутствие данных соответствующих клинических исследований, по аналогии с ХОБЛ, эксперты рекомендуют своевременно включать пациентов с ЛАМ в программы по легочной реабилитации и проводить противогриппозную и пневмококковую вакцинацию [2].

\section{Литература}

1. Clements D., Chang W.Y.C., Johnson S.R. Lymphangioleiomyomatosis. Eur Respir. Mon. 2009; 46: 176-207.

2. European Respiratory Society guidelines for the diagnosis and management of lymphangioleiomyomatosis. Eur. Respir. J. 2010; 35: 14-26.

3. von Stossel E. Uber muskulare Cirrhose der Lunge [Muscular cirrhosis of the lungs]. Beitr. Klin. Tuberk. 1937; 90: 432-442.

4. Urbun T., Lozor R., Lacronique J. et al. Pulmonary lymphangioleiomyomatosis. A study of 69 patients. Group d'Etudes et de Recherche sur les Maladies "Orphelines" Pulmonaires (GERM "O"P) [Study and research group on orphan lung disease]. Medicine (Baltimore) 1999; 78 (5): 321-337.

5. Johnson S.R., Taffersfield A.E. Decline in lung function in lymphangioleiomyomatosis: relation to menopause and progesterone treatment. Am. J. Resrir. Crit. Care Med. 1999; 160 (2): 628-633.

6. Moss J., Avili N.A., Barnes P.M. et al. Prevalence and clinical characteristics of lymphangioleiomyomatosis (LAM) in patients with tuberous sclerosis complex. Am. J. Respir. Crit. Care Med. 2001; 164 (4): 669-671.

7. Yates J.R.W. Tuberous sclerosis. Eur. J. Hum. Genet. 2006; 14 (10): 1065-1073.

8. Osborne J.P., Fryer A., Webb D. Epidemiology of tuberous sclerosis. Ann. N. Y. Acad. Sci.1991; 615: 125-128.

9. Lutembacher R. Dyesmbryomes metatypiques des reinds; carcinose submallaire aigue du puomon avec emphseme generalise et double pneumothorax [Atypical kidney dysembryomas: acute submiliary carcinomatosis of the lung with emphysema and double pneumothorax]. Ann. Med. 1918; 5: 435-450.

10. Hancock E., Tomkins S., Sampson J. et al. Lymphangioleiomyomatosis and tuberous sclerosis. Respir. Med. 2002; 96: $7-13$.

11. Castro M., Shepherd C.W., Gomez M.R. et al. Pulmonary tuberous sclerosis. Chest 1995; 107: 189-195.
12. Johnson S.R., Taffersfield A.E. Clinical experience of lymphangioleiomyomatosis in the UK. Thorax 2000; 55 (12): 1052-1057.

13. Oh Y.M., Mo E.K., Jang S.H. et al. Pulmonary lymphangioleiomyomatosis in Korea. Thorax 1999; 54: 618-621.

14. Hayashida M., Seyama K., Inoue Y. et al. The epidemiology of lymphangioleiomyomatosis in Japan: a nationwide crosssectional study of presenting features and prognostic factors. Respirology 2007; 12: 523-530.

15. Carsillo T., Astrinidis A., Henske E.P. Mutations in the tuberous sclerosis complex gene TSC2 are a cause of sporadic pulmonary lymphangioleiomyomatosis. Proc. Natl. Acad. Sci. USA 2000; 97 (11): 6085-6090.

16. Чучалин А.Г. (ред.) Респираторная медицина. Руководство. М.; 2007; т. 2: 303-311.

17. Crooks D., Pacheco-Rodriguez G., DeCastro R. et al. Molecular and genetic analysis of disseminated neoplastic cells in lymphangioleiomyomatosis. Proc. Natl. Acad. Sci. USA 2004; 101: 17462-17467.

18. Hirama M., Atsura R., Mitani K. et al. Lymphangioleiomyomatosis diagnosed by immunocytochemical and genetic analysis of lymphangioleiomyomatosis cell clusters found in chylous pleural effusion. Intern. Med. 2007; 46: 1593-1596.

19. Sato T., Seyama K., Fujii H. et al. Mutation analysis of TSC1 and TSC2 genes in Japanese patients with pulmonary lymphangioleiomyomatosis. J. Hum. Genet. 2002; 47 (1): 20-28.

20. Goncharova E.A., Goncharov D.A., Eszterhas A. et al. Tuberin regulates p70S6 kinase activation and ribosomal protein S6 phosphorylation. A role for the TSC2 tumor suppressor gene in pulmonary lymphangioleiomyomatosis (LAM). J. Biol. Chem. 2002; 277 (34): 30958-30967.

21. Pan C., Chung M., Ng K. et al. Constant allelic alteration on chromosome $16 \mathrm{p}$ (TSC2 gene) in perivascular epithelioid cell tumor (PEComa): genetic evidence for the relationship of PEComa with angiomyolipoma. J. Pathol. 2008; 214: 387-393.

22. Pea M., Bonetti F., Zamboni G. et al. Clear cell tumor and angiomyolipoma. Am. J. Surg. Pathol. 1991; 15: 199-202.

23. Абелев Г.И., Эрайзер Т.Р. На пути к пониманию природы рака. Биохимия 2008; 73 (5): 605-618.

24. Seyama K., Kumasaka T., Souma S. et al. Vascular endothelial growth factor-D in increased in serum of patients with lymphangioleiomyomatosis. Lymphat. Res. Biol. 2006; 4: 143-152.

25. Matsui K., Takeda K., Yu Z.X. et al. Role for activation of matrix metalloproteinases in the pathogenesis of pulmonary lymphangioleiomyomatosis. Arch. Pathol. Lab. Med. 2000; 124: 267-275.

26. Zhe X., Yang Y., Jakkaraju S. et al. Tissue inhibitor of metalloproteinase-3 downregulation in lymphangioleiomyomatosis: potential consequence of abnormal serum response factor expression. Am. J. Respir. Cell. Mol. Biol. 2003; 28: 504-511.

27. Клишко Е.В., Кондакова И.В., Чойнзонов Е.Л. Матриксные металлопротеиназы в онкогенезе. Сиб. онкол. журн. 2003; 2: 63-70.

28. Henry M.T., McMahol K., Mackarel A.J. et al. Matrix metalloproteinases and inhibitor of metalloproteinase-1 in sarcoidosis and IPF. Eur. Respir. J. 2002; 20: 1220-1227.

29. Henske E.P. Metastasis of benign tumor cells in tuberous sclerosis complex. Genes Chromosom. Cancer 2003; 38: 376-381.

30. Karbowniczek M., Astrinidis A., Balsara B.R. et al. Recurrent lymphangioleiomyomatosis after transplantation: genetic analyses reveal a metastatic mechanism. Am. J. Respir. Crit. Care Med. 2003; 167: 976-982. 
31. Johnson S. Rare diseases. 1. Lymphangioleiomyomatosis: clinical features, management and basic mechanisms. Thorax 1999; 54 (3): 254-264.

32. Ryu J.H., Moss J., Beck G.J. et al. The NHLBI lymphangioleiomyomatosis registry: characteristics of 230 patients at enrollment. Am. J. Respir. Crit. Care Med. 2006; 173 (1): 105-111.

33. Cohen M.M., Pollock-BarZiv S., Johnson S.R. Emerging clinical picture of lymphangioleiomyomatosis. Thorax 2005; 60: 875-879.

34. Sinclar W., Wright J.L., Churg A. Lymphangioleiomyomatosis presenting in postmenopausal women. Thorax 1985; 40: 75-76.

35. Logan R.F., Fawcett I.W. Oophorectomy for pulmonary lymphangioleiomyomatosis: a case report. Br. J. Dis. Chest 1985; 79: 98-100.

36. Tomasien A., Greenberg M.S., Rumerman H. Tamoxifen for lymphangioleiomyomatosis. N. Engl. J. Med. 1982; 306: 745-746.

37. Glassberg M.K., Elliot S.J., Fritz J. et al. Activation of the estrogen receptor contributes to the progression of pulmonary lymphangioleiomyomatosis via MMP-induced cell invasiveness. J. Clin. Endocrinol. 2008; 93 (5): 1625-1633.

38. Matsui K., Takeda K., Yu Z.X. et al. Downregulation of estrogen and progestorone receptors in the abnormal smooth muscle cells in pulmonary lymphangioleiomyomatosis following therapy. An immunohistochemical study. Am. J. Respir. Crit. Care Med. 2000; 161 (3, Pt 1): 1002-1009.

39. Каменева М.Ю., Двораковская И.В., Новикова Л.Н. и др. Лейомиоматоз легких (Морфофункциональное исследование). Болезни органов дыхания 2007; 1: 86-95.

40. Miyake M., Tateishi U., Maeda T. et al. Pulmonary lymphangioleiomyomatosis in a male patient with tuberous sclerosis complex. Radiat. Med. 2005; 23: 525-527.

41. Aubry M.-C., Myers J.L., Ryu J.H. et al. Pulmonary lymphangioleiomyomatosis in a man. Am. J. Respir. Crit. Care Med. 2000; 162: 749-752.

42. Fiore M.G., Sanguedolce F., Lolli I. et al. Abdominal lymphangioleiomyomatosis in a man with Klinefelter syndrome: the first reported case. Ann. Diagn. Pathol. 2005; 9: 96-100.

43. Schiavina M., Di Scioscio V., Contini P. et al. Pulmonary lymphangioleiomyomatosis in a karyotypically normal man without tuberous sclerosis complex. Am. J. Respir. Crit. Care Med. 2007; 176: 96-98.

44. Sullivan E.J. Lymphangioleiomyomatosis: a review. Chest 1998; 114 (6): 1689-1703.

45. Перепечин В., Стеблянская О., Черняев А. и др. Диффузный лимфангиолейомиоматоз легких. Врач 2008; 2: $6-11$.

46. Chu S.C., Horiba K., Usuki J. et al. Comprehensive evaluation of 35 patients with lymphangioleiomyomatosis. Chest 1999; 115: 1041-1052.

47. Almoosa K.F., Ryu J.H., Mendez J. et al. Management of pneumothorax in lymphangioleiomyomatosis: effects on recurrence and lung transplantation complications. Chest 2006; 129 (5): 1274-1281.

48. Ryu J.H., Doerr C.H., Fisher S.D. et al. Chylothorax in lymphangioleiomyomatosis. Chest 2003; 123: 623-627.

49. Corrin B., Liebow A.A. Friedman P.J. Pulmonary lymphangioleiomyomatosis. Am. J. Pathol. 1975; 79: 348-382.

50. Matsui K., Tatsuguchi A., Valencia J. et al. Extrapulmonary lymphangioleiomyomatosis (LAM): clinicopathologic features in 22 cases. Hum. Pathol. 2000; 31 (10): 1242-1248.
51. Avila N.A., Bechtle J., Dwyer A.J. et al. Lymphangioleiomyomatosis: CT of diurnal variation of lymphangioleiomyomas. Radiology 2001; 221 (2): 415-421.

52. Avila N.A., Kelly J.A., Chu S.C.et al. Lymphangioleiomyomatosis: abdominopelvic CT and US findings. Radiology 2000; 216 (1): 147-153.

53. Peh W.C., Law S., Fok M. et al. Case report: lymphangioleiomyomatosis with spontaneous peritoneal rupture. Br. J. Radiol. 1994; 67 (798): 605-608.

54. Gray S., Carrington C., Cornog J. Lymphangioleiomyomatosis: report of case with ureteral involvement and chyluria. Cancer (Phclad.) 1975; 35: 490-498.

55. Maziak D.E., Kesten S., Rappaport D.S. et al. Extrathoracic angiomyolipomas in lymphangioleiomyomatosis. Eur. Respir. J. 1996; 9: 402-405.

56. Bernstein S.M., Newell J.D., Adamczyk D. et al. How common are renal angiomyolipomas in patients with pulmonary lymphangioleiomyomatosis? Am. J. Respir. Crit. Care Med. 1995; 152: 2138-2143.

57. O'Callaghan F.J., Noakes M.J., Martyn C.N. et al. An epidemiological study of renal pathology in tuberous sclerosis complex. Br. J. Urol. International 2004; 94 (6): 853-857.

58. van Baal J.G., Smits N.J., Keeman J.N. et al. The evolution of renal angiomyolipomas in patients with tuberous sclerosis complex [comment]. J. Urol. (Baltimore) 1994; 152 (1): $35-38$.

59. Ewalt D.H., Sheffield E., Sparagana S.P. et al. Renal lesion growth in children with tuberous sclerosis complex. J. Urol. (Baltimore) 1998; 160 (1): 141-145.

60. Roach E.S., DiMario F.J., Kandt R.S. et al. Tuberous Sclerosis Consensus Conference: recommendations for diagnostic evaluation. National Tuberous Sclerosis Association. J. Child Neurol. 1999; 14 (6): 401-407.

61. Rakowski S.K., Winterkorn E.B., Paul E. et al. Renal manifestations of tuberous sclerosis complex: Incidence, prognosis, and predictive factors. Kidney Int. 2006; 70 (10): 1777-1782.

62. Lemaitre L., Robert Y., Dubrulle F. et al. Renal angiomyolipoma: growth followed up with CT and / or US. Radiology 1995; 197 (3): 598-602.

63. Avila N.A., Dwyer A.J., Rabel A. et al. Sporadic lymphangioleiomyomatosis and tuberous sclerosis complex with lymphangioleiomyomatosis: comparison of CT features. Radiology 2007; 242: 277-285.

64. Warth A., Herpel E., Schmahl A. et al. Mediastinal angiomyolipomas in a male patient affected by tuberous sclerosis. Eur. Respir. J. 2008; 31: 678-680.

65. L'Hostis H., Deminiere C., Ferriere J.M. et al. Renal angiomyolipoma: clinicopathologic, immunohistochemical, and follow-up study of 46 cases. Am. J. Surg. Pathol. 1999; 23: 1011-1020.

66. Steiner M.S., Goldman S.M., Fishman E.K. et al. The natural history of renal angiomyolipoma. J. Urol. (Baltimore) 1993; 150: 1782-1786.

67. Moss J., DeCastro R., Patronas N.J. et al. Meningiomas in lymphangioleiomyomatosis. J.A.M.A. 2001; 286 (15): 1879-1881.

68. Bonetti F., Chiodera P.L., Pea M. et al. Transbronchial biopsy in lymphangioleiomyomatosis of the lung. HMB-45 for diagnosis. Am. J. Surg. Pathol. 1993; 17 (11): 1092-1102.

69. Matsui K., Beasley M.B., Nelson W.K. et al. Prognostic significance of pulmonary lymphangioleiomyomatosis histologic score. Am. J. Surg. Pathol. 2001; 25 (4): 479-484.

70. Carrington C.B., Cugell D.W., Gaensler E.A. et al. Lymphangioleiomyomatosis. Physiologic-pathologic-radiologic correlations. Am. Rev. Respir. Dis. 1977; 116 (6): 977-995. 
71. Matsumoto Y., Horiba K., Usuki J. et al. Markers of cell proliferation and expression of melanosomal antigen in lymphangioleiomyomatosis. Am. J. Respir. Cell Mol. Biol. 1999; 21 (3): 327-336.

72. Zhe X., Schuger L. Combined smooth muscle and melanocytic differentiation in lymphangioleiomyomatosis. J. Histochem Cytochem 2004; 52 (12): 1537-1542.

73. Franz D.N., Brody A., Meyer C. et al. Mutational and radiographic analysis of pulmonary disease consistent with lymphangioleiomyomatosis and micronodular pneumocyte hyperplasia in women with tuberous sclerosis. Am. J. Respir. Crit. Care Med. 2001; 164 (4): 661-668.

74. Taveira-DaSilva A.M., Stylianou M.P., Hedin C.J. et al. Decline in lung function in patients with lymphangioleiomyomatosis treated with or without progesterone. Chest 2004; 126 (6): 1867-1874.

75. Lazor R., Valeyre D., Lacronique J. et al. Low initial KCO predicts rapid FEV1 decline in pulmonary lymphangioleiomyomatosis. Respir. Med. 2004; 98 (6): 536-541.

76. Fujimoto M., Ohara N., Sasaki H. et al. Pregnancy complicated with pulmonary lymphangioleiomyomatosis: case report. Clin. Exp. Obstetr. Gynecol. 2005; 32 (3): 199-200.

77. Brunelli A., Catalini G., Fianchini A. Pregnancy exacerbating unsuspected mediastinal lymphangioleiomyomatosis and chylothorax. Int. J. Gynaecol. Obstet. 1996; 52 (3): 289-290.

78. McLoughlin L., Thomas G., Hasan K. Pregnancy and lymphangioleiomyomatosis: anaesthetic management. Int. J. Obstetr. Anest. 2003; 12 (1): 40-44.

79. Yockey C.C., Riepe R.E., Ryan K. Pulmonary lymphangioleiomyomatosis complicated by pregnancy. Kans. Med. 1986; 87 (10): 277-278, 293.

80. Warren S.E., Lee D., Martin V., Messink W. Pulmonary lymphangiomyomatosis causing bilateral pneumothorax during pregnancy. Ann. Thorac. Surg. 2000; 55 (4): 998-1000.

81. Raft J., Lalot J.M., Meistelman C., Longrois D. Renal angiomyolipoma rupture during pregnancy. Gynecol. Obstetr. Fertil. 2006; 34 (10): 917-919.

82. Storm D.W., Mowad J.J. Conservative management of a bleeding renal angiomyolipoma in pregnancy. Obstet. \& Gynecol. 2006; 107 (2, Pt 2): 490-492.

83. Morales J.P., Georganas M., Khan M.S. et al. Embolization of a bleeding renal angiomyolipoma in pregnancy: case report and review. Cardiovasc. Intervent. Radiol. 2005; 28 (2): 265-268.

84. Mascarenhas R., McLaughlin P. Haemorrhage from angiomyolipoma of kidney during pregnancy - a diagnostic dilemma. Irish Med. J. 2001; 94 (3): 83-84.

85. Pollock-BarZiv S., Cohen M.M., Downey G.P. et al. Air travel in women with lymphangioleiomyomatosis. Thorax 2007; 62 (2): 176-180.

86. Avila N.A., Dwyer A.J., Rabel A. et al. CT of pleural abnormalities in lymphangioleiomyomatosis and comparison of pleural findings after different types of pleurodesis. Am. J. Roentgenol. 2006; 186 (4): 1007-1012.
87. Wójcik P., Otto T., Jagiello R. et al. Use of pleuro-peritoneal shunt in the treatment of chronic chylothorax. Pneumonol. Alergol. Pol. 1998; 66: 473-479.

88. Taylor J.R., Ryu J., Colby T.V., Raffin.TA. Lymphangioleiomyomatosis. Clinical course in 32 patients. N. Engl. J. Med. 1990; 323 (18): 1254-1260.

89. Svendsen T.L., Viskum K., Hansborg N. et al. Pulmonary lymphangioleiomyomatosis: a case of progesterone receptor positive lymphangioleiomyomatosis treated with medroxyprogesterone, oophorectomy and tamoxifen. Br. J. Dis. Chest 1984; 78 (3): 264-271.

90. Morimoto N., Hirasaki S., Kamei T. et al. Pulmonary lymphangiomyomatosis (LAM) developing chylothorax. Intern Med 2000; 39(9): 738-741.

91. Christodoulou M., Ris H.-B., Pezzetta E. Video-assisted right supradiaphragmatic thoracic duct ligation for non-traumatic recurrent chylothorax. Eur. J. Cardiothorac Surg. 2006; 29 (5): 810-814.

92. Druelle.S, Aubry P., Levi-Valensi P. Pulmonary lymphangiomyomatosis: a 3-year followup of medroxyprogesterone acetate therapy. Apropos of a case. Rev. Pneumol. Clin. 1995; 51 (5): 284-287.

93. Zanella A., Toppan P., Nitti D., Lise M. Pulmonary lymphangioleiomyomatosis: a case report in postmenopausal woman treated with pleurodesis and progesterone (medroxyprogesterone acetate). Tumori 1996; 82 (1): 96-98.

94. Yamakado K., Tanaka N., Nakagawa T. et al. Renal angiomyolipoma: relationships between tumor size, aneurysm formation, and rupture. Radiology 2002; 225 (1): 78-82.

95. Taveira-DaSilva A.M., Hedin C., Stylianou M.P. et al. Reversible airflow obstruction, proliferation of abnormal smooth muscle cells, and impairment of gas exchange as predictors of outcome in lymphangioleiomyomatosis. Am. J. Respir. Crit. Care Med. 2001; 164 (6): 1072-1076.

96. Harari S., Cassandro R., Chiodini J. et al. Effect of a gonadotrophin-releasing hormone analogue on lung function in lymphangioleiomyomatosis. Chest 2008; 133 (2): 448-454.

97. Bissler J.J., McCormack F.X., Young L.R. et al. Sirolimus for angiomyolipoma in tuberous sclerosis complex or lymphangioleiomyomatosis. N. Engl. J. Med. 2008; 358 (2): $140-151$.

98. Davies D.M., Johnson S.R., Tattersfield A.E. et al. Sirolimus therapy in tuberous sclerosis or sporadic lymphangioleiomyomatosis. N. Engl. J. Med. 2008; 358 (2): 200-203.

\section{Информация об авторах}

Овчаренко Светлана Ивановна - д. м. н., проф. кафедры факультетской терапии № 1 лечебного факультета ГОУ ВПО ММА им. И.М.Сеченова; тел. / факс: (499) 248-56-67; e-mail: svetftk@mail.ru

Сон Елена Алексеевна - к. м. н., ассистент кафедры факультетской терапии № 1 лечебного факультета ГОУ ВПО ММА им. И.М. Сеченова; тел. / факс: (499) 248-45-23; e-mail: sonalex1110@mail.ru 